\title{
Tumeur mixte épithéliale et stromale maligne: A propos d'un nouveau cas chez l'homme révélé par une pyonéphrose sur lithiase coralliforme
}

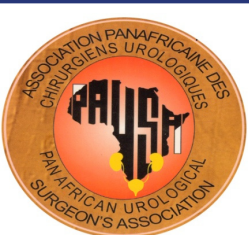

B. Sine $^{1}$, A.M. Gaye ${ }^{2}$, N.A. Bagayogo ${ }^{1}$, A. Sarr ${ }^{1}$, Gaye ${ }^{l}$, Ndiaye $M^{1}$, N.S. Ndour ${ }^{1}$, Ze Ondo C. ${ }^{1}$, A.

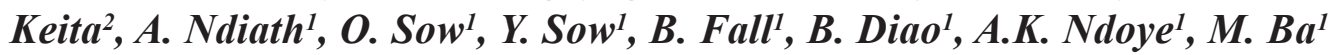

${ }^{I}$ Service d'Urologie Andrologie du CHU Aristide Le Dantec, ${ }^{2}$ Service d'Anatomie Pathologique du CHU Aristide Le Dantec

\section{Résumé}

Introduction: Les tumeurs rénales mixtes épithéliale et stromale (TMES) constituent une nouvelle entité dans la classification OMS des tumeurs du rein. Nous rapportons un cas de TMES maligne chez un homme de 32 ans révélée par une pyonéphrose sur lithiase rénale coralliforme.

Observation: Il s'agissait d'un patient de 32 ans adressé pour une prise en charge d'une masse rénale et d'une altération de l'état général. L'examen avait conclu à une pyonéphrose sur lithiase rénale droite coralliforme. L'examen anatomo-pathologique de la pièce de néphrectomie avait mis en évidence une TMES maligne. L'évolution a été marquée par la survenue de métastases et le décès du patient.

Conclusion: La TMES maligne est une entité rare. Du fait de sa potentielle agressivité, des critères diagnostiques doivent être mis sur pied afin d'avoir un traitement bien codifié.

Mots clés: Rein; tumeur mixed épithéliale et stromale; chirurgie.

Rec, u le: 14 October 2019, Accepté le: 24 January 2020

Auteur correspondant: Babacar Sine, Service d'Urologie Andrologie du CHU Aristide Le Dantec,

Email: papesine@yahoo.fr

ISSN: 2090-2379, https://afju.journals.ekb.eg

\section{Introduction}

La tumeur mixte épithéliale et stromale (TMES) du rein est une entité rare. Elle représente 0, 2\% des cancers rénaux ${ }^{[1]}$. Elle a été individualisée en 1998 et décrite dans la classification de l'organisation Mondiale de la Santé (OMS) six ans plus tard ${ }^{[2-3]}$. Elle se rencontre surtout chez la femme en période péri-ménopausique avec des antécédents de traitement par oestro-progestatifs ou de chirurgie gynécologique. L'association TMSE et lithiase n'a jamais été décrite. L'objectif de ce manuscrit était de rapporter une TMES maligne chez un homme ayant consulté pour la prise en charge d'une pyonéphrose sur lithiase rénale coralliforme.

\section{Observation}

M. S était un patient de 32 ans, asthmatique connu et suivi. Il a été adressé pour la prise en charge d'une masse abdominale et une altération de l'état général évoluant depuis plusieurs mois. L'examen avait mis en évidence une fièvre, une anémie clinique, une déshydration et une dénutrition, un gros rein gauche, fixe, ferme, s'étendant de l'hypochondre gauche jusqu'à la crête iliaque homolatérale. A la biologie il y avait une créatininémie normale, une hyperleucocytose à 13700 éléments/mm3 et une anémie à $7,9 \mathrm{~g} / \mathrm{dl}$. L'Uro-Tomodensitométrie (Uro-TDM) avait révélé une lithiase rénale coralliforme gauche responsable d'une dilatation pyélo-calicielle avec absence de sécrétion et d'excrétion du produit de contraste. Le diagnostic d'une pyonéphrose a été alors retenu. Une néphrostomie gauche a été ainsi réalisée avec drainage d'environ 1, 5 litres de pus franc dont la culture était stérile. L'évolution étant marquée par une persistance de la masse rénale, l'indication d'un drainage plus ou moins néphrectomie par voie ouverte a été retenue. A l'exploration, il s'agissait d'une pyonéphrose avec de multiples lithiases pyéliques et calicielles. Une néphrectomie a été ainsi réalisée malgré l'adhérence du pôle inferieur du rein au muscle psoas (Figure 1). Une antibiothérapie parentérale puis orale à base de Céfotaxime a été administrée avec des transfusions de 
sang total. Les suites opératoires étaient simples. L'examen anatomo-pathologique de la pièce opératoire avait mis en évidence une tumeur mixte épithéliale et mésenchymateuse envahissant tout le parenchyme rénal, le hile et la graisse péri-rénale sans embole tumorale. L'examen immunohistochimique (Figure 2) avait montré Pancytokératine
+, CK7-, CD10+, EMA+, Desmine-, Vimentine+, Bcl2-, $\mathrm{CD} 99+, \mathrm{RO}+, \mathrm{RP}+$. Un mois plus tard le patient est reçu pour altération de l'état général et masse épigastrique. La TDM avait révélé des lésions hépatiques gauches s'accordant avec des métastases. Le patient est décédé dans un tableau de cachexie.

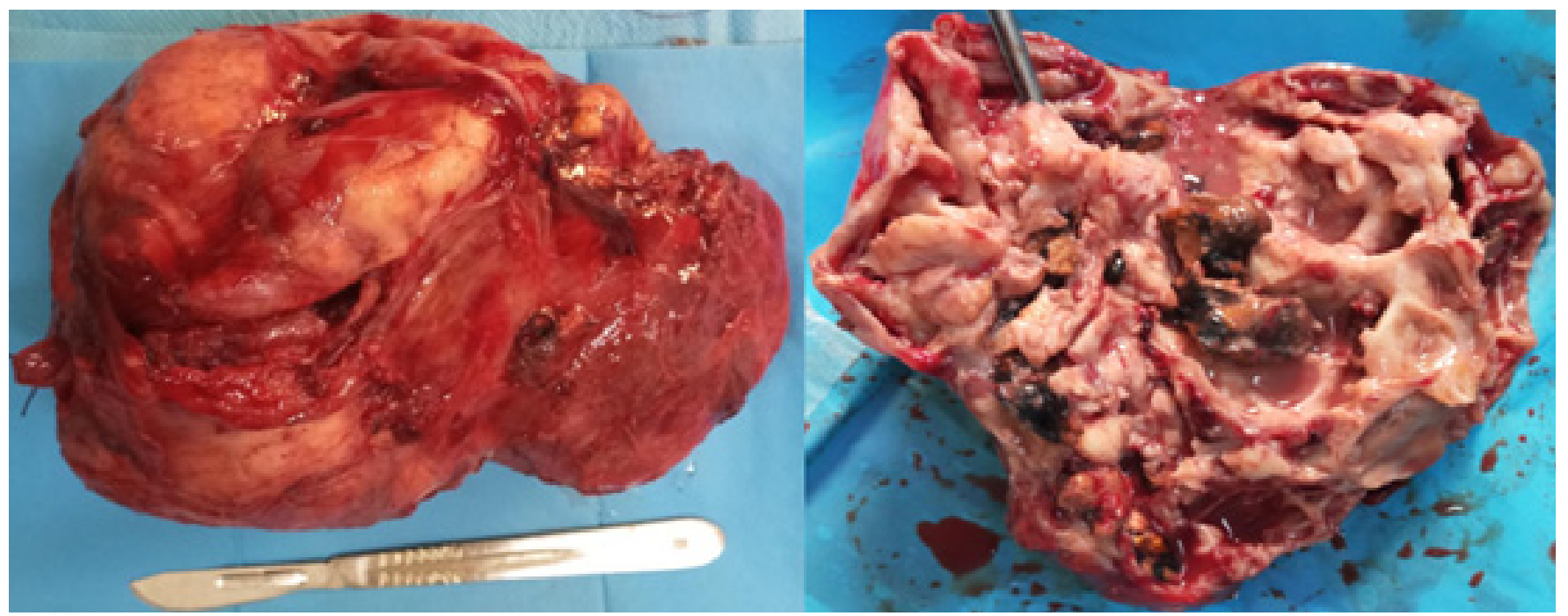

Fig. 1: pièce de néphrectomie totale $(\mathrm{A})$ et aspect du rein après ouverture montrant du pus et des lithiases pyéliques et calicielles (B).

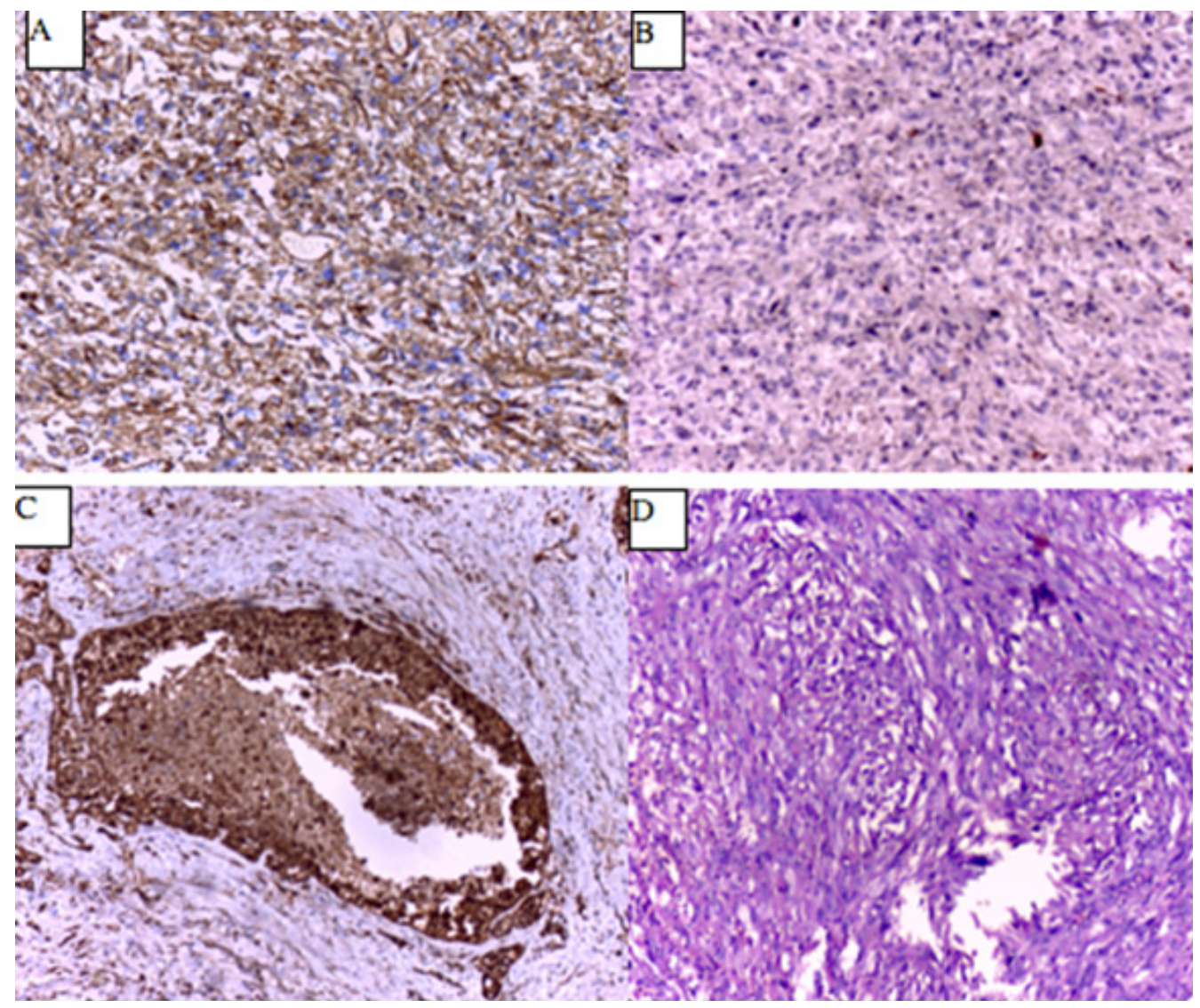

Fig. 2: Aspect histologique et immuno-histochimique

A : CD99+ marquage des cellules conjonctives ( $x 100)$

$\mathrm{B}$ : Pancytokératine négative sur la composante conjonctive (x100)

C : Pancytokératine positive sur la composante épithéliale (x100)

D : Composante conjonctive HE (x100) 


\section{Discussion}

La tumeur mixte épithéliale et stromale (TMES) est une entité récemment introduite dans le classification de l'Organisation Mondiale de la Santé de 2004[3]. La transformation maligne est rare, jusqu'à maintenant 13 cas avaient été décrits dans la littérature dont trois chez $l^{\prime} h o m m e^{[4]}$. La pathogénie n'est pas toujours connue. L'imprégnation oestro-progestative y jouerait un rôle. Ce qui explique sa fréquence chez la femme et sa rareté chez l'homme. Cependant même chez l'homme ces TMES expriment les récepteurs oestro-progestatifs (RO et RP) à l'immuno-histochimie comme c'est le cas chez notre patient.

L'association de cette tumeur à la lithiase urinaire est rapportée pour la première fois. Toutefois nous n'avons pas réussi à identifier le lien qui existerait entre le développement de ces tumeurs et la lithogénèse.

La symptomatologie des tumeurs rénales est souvent faite de douleurs lombaires, masse rénale, et hématurie. Pour les patients qui consultent tardivement il n'est pas rare d'avoir une anémie clinique et une altération de l'état général comme ce fut le cas chez notre patient. Cette altération de l'état général était due d'abord à la pyonéphrose qu'avait eue le patient et ensuite probablement au cancer avancé en dessous.

La TDM est passée à côté du diagnostic. En effet l'aspect radiologique typique de cette tumeur est une masse kystique intra-parenchymateuse ou intra-sinusale évoquant un néphrome kystique ou un carcinome rénal à cellules claires $^{[5-6]}$. La lithiase coralliforme et la dilatation des cavités rénales ont sans doute plus attiré l'attention du radiologue.

La chirurgie carcinologique obéit à des règles bien déterminées. La néphrectomie réalisée pour notre patient était dans le but de traiter la pyonéphrose. De ce fait certaines mesures élémentaires de la chirurgie carcinologique n'ont pas été respectées comme la ligature première du pédicule. Cela pourrait expliquer en partie la récidive de la tumeur avec des métastases hépatiques. Du fait de la rareté de ce type histologique, le traitement n'est pas encore codifié. Il associerait une chirurgie, une chimiothérapie ou une radiothérapie selon les centres ${ }^{[7-8]}$. Les données sur le traitement systémique sont limitées, mais certaines TMES malignes seraient chimio-sensibles et répondraient à la doxorubicine et à l'ifosfamide ${ }^{[4]}$. L'utilisation des antiangiogéniques et l'immunothérapie dans le traitement de ces cancers n'as pas été rapportée.

Le pronostic des TMES malignes est défavorable car elles constituent des tumeurs agressives. L'évolution après traitement est souvent marquée par les récidives et les métastases $^{[4-7]}$.

\section{Conclusion}

La TMES maligne est une entité rare. Du fait de sa potentielle agressivité, des critères diagnostiques doivent être mis sur pied afin d'avoir un traitement bien codifié. L'association d'une lithiase rénale à une TMES maligne est bien possible. D'où la nécessité d'augmenter la vigilance chez les radiologues pour ne pas passer à côté d'une TMES maligne dont seule la précocité du diagnostic peut donner des chances de guérison au patient.

\section{Intérêts concurrents}

Les auteurs déclarent ne pas avoir de conflits d'intérêts en relation avec cet article.

\section{Ethique}

Le consentement éclairé des parents a été obtenu pour l'utilisation des images et l'exploitation du dossier médical à des fins scientifiques.

\section{Contribution Des Auteurs}

Dr Abdou Magib Gaye, Pr Y. Sow: ont activement participé à la rédaction et à la correction de l'article.

Dr Alioune Sarr, Dr Ndeye Aissatou Bagayogo, Dr Omar Gaye, Dr Modou Ndiaye, Dr Ndiaga Seck Ndour $^{1}$, Dr Cyrille Ze Ondo, Dr Abdoulaye Keita ${ }^{2}$, Dr A. Ndiath, Dr O. Sow: et nous avons collaboré ensemble dans la prise en charge du patient, la rédaction et la correction de ce document.

Les professeurs Boubacar Fall, Babacar Diao, Alain K. Ndoye, Mamadou Ba: sont les responsables d'enseignement dans notre service. Ils ont tous contribué à la rédaction et la correction de cet article.

Source of funding: Aucun financement

\section{Références}

1. Terao H, Makiyama K, Yanagisawa M, Miyake M, Sano F, Kita K, et al. Mixed epithelial and stromal tumor of kidney: a case report. Hinyokika Kiyo. 2009; 55: 495-8.

2. Michal M, Syrucek M. Benign mixed epithelial and stromal tumor of the kidney. Pathol Res Pract. 199; 8194: 445-8.

3. Eble JN. Mixed epithelial and stromal tumour. In:Eble JN, Sauter G, Epstein JI, Sesterhenn IA, editors. World Health Organisation classification of 
tumours. Pathology and genetics of tumours of the urinary system and male genital organs. Lyon : IARC Press; 2004. p. 239-45

4. Yap YS, Coleman M, Olver I. Aggressive mixed epithelial stromal tumor of the kidney treated with chemotherapy and radiotherapy. Lancet Oncol 2004;5:747-9.

5. Smichi I, Zehani A, Chelly I, Khadhar A, Azouz H, Nouira Y et al. À propos d'une tumeur rarissime du rein: la tumeur mixte épithéliale et stromale maligne. Progrès en urologie 2015, 25(6), 293-7.
6. Battisti S, Renaudin K, Rigaud J, Hetet JF, Dugardin F, Le Normand L, et al. Mixed epithelial and stromal tumour of the kidney. Prog Urol 2004;14:210-2

7. Bex A, Albiges L, Ljungberg B, Bensalah K, Dabestani $\mathrm{S}$, Giles RH, et al. Updated European Association of Urology Guidelines for Cytoreductive Nephrectomy in Patients with Synchronous Metastatic Clear-cell Renal Cell Carcinoma. Eur Urol 2018; 74(6):805-9

8. Bakavičius A, Barisienė M, Snicorius M, Valanciené $\mathrm{D}$, Dasevicius D, Zalimas A, et al. Malignant mixed epithelial and stromal tumour of the kidney:a case report and a literature review. Acta Med Litu 2018;25(1):31-7. 


\title{
Malignant mixed epithelial and stromal tumor: About a new case in man revealed by pyonephrosis on staghorn calculi
}

\begin{abstract}
Introduction: Mixed epithelial and stromal renal tumors (MEST) represent a new entity in the WHO classification of tumors of the kidney.

Observation: We report a case of malignant MEST in a 32 years old man revealed by pyonephrosis on staghorn nephrolithiasis. Observation A 32 years old patient was addressed for a management of a renal mass and a deterioration of the general condition. The exams had found a pyonephrosis right staghorn nephrolithiasis. Anatomo-pathological examination of the nephrectomy specimen revealed a malignant MEST. The evolution was marked by the occurrence of metastases and the death of the patient. Conclusion: Malignant MEST is a rare entity. Because of its potential aggressiveness, diagnostic criteria must be established in order to have a well-coded treatment.
\end{abstract}

Keywords: Kidney, mixed epithelial and stromal tumor, surgery. 